\title{
Immunofluorescent detection of Tie1 in the endothelium of the Rat and Human corpus cavernosum during aging
}

\author{
J.A. Fonseca*, I. Tomada***, N. Tomada**,***, H. Almeida**** and D. Neves**** \\ *Department of Experimental Biology, Faculty of Medicine of Universidade do Porto, Alameda Prof. \\ Hernâni Monteiro, 4200-319 Porto, Portugal \\ **Instituto de Biologia Molecular e Celular (IBMC) of Universidade do Porto, Rua do Campo Alegre 823, \\ 4150-180 Porto, Portugal \\ ***Department of Urology, Central Hospital of S. João- Alameda Prof. Hernâni Monteiro, 4200-319 \\ Porto, Portugal
}

Vasculogenic erectile dysfunction (ED) is an age-related disease partially dependent on vascular growth factors availability and their specific endothelium receptors activation. Among these, angiopoietinstyrosine kinase with immunoglobulin and EGF homology domain-2 (Tie2) system seems to be particularly dependent on aging, both in rat and in human corpus cavernosum (CC) [1,2]. Angiopoietins (Ang) 1 and 2 are produced by pericytes and endothelial cells respectively, and bind with the same affinity to Tie 2 receptor. Ang1 was shown to act as an obligatory agonist inducing Tie2 phosphorylation, which promotes cell-cell and cell-extracellular matrix interactions. On the other hand, Ang2 acts in a context-dependent fashion in the increment or, otherwise, destabilization of pre-existent vasculature [3].

Interestingly, it has been demonstrated that activation of Tie 2 receptor could also be modulated by Tie1, an additional member of the Tie family of receptor tyrosine kinases [4], and that co-expression of Tie2 is required for Tie1 activation. Indeed, Tie1 similarly to Tie2 is involved in the maintenance of the vascular integrity [5]. Nevertheless, its role in Angiopoietins-Tie system regulation remains poorly understood, and the identification of a true ligand has remained elusive. In the present study, we aim to demonstrate the expression of Tie1 in the endothelium of aged CC in the Rat and Human species.

Twenty-five male Wistar rats were divided in five groups $(\mathrm{n}=5)$ and sacrificed by decapitation when they reached the ages of study $(6,12,18,24$ and 36 months). The penises were excised and immediately fixed in formalin solution. Human CC fragments were obtained from organ donors without known risk factors for ED and divided in two groups: young (16-35 years) and aged (59-74 years). Paraffin embedded sections were deparaffinized, rehydrated and submitted to epitope retrieval with heated Tris-EDTA pH 9.0 buffer before dual-immunolabeling of Tie1 (rabbit anti-Tiel antibody -abcam) and specific markers of endothelium and smooth muscle cell (goat- anti-PECAM-1 and mouse anti- $\alpha$-actin antibodies, Santa Cruz Biotechnology Inc. and Millipore, respectively). Appropriate secondary antibodies were employed (antirabbit labelled with Alexa 488 with anti-mouse, or, anti -goat labelled with Alexa 568). Nuclei counterstaining was achieved with 4'-6-diamino-2-phenylindole. Images were observed in an Apotome microscope (Carl Zeiss System, Göttingen, Germany) using the filter sets, 01 to DAPI, 09 to Alexa 488 and 31 to Alexa 568, and acquired with Axio Vision 3.0 program (Carl Zeiss System).

Tie1 was detected in the endothelium in the CC of Rat, co-localizing with PECAM-1 (Figure 1a) but not with $\alpha$-actin (Figure 1b). The intensity of Tie1 immunolabeling was higher in the older animals (24 and 36 months). Tie1 was also detected in Human CC endothelium, suggesting an up-regulation in samples obtained from older individuals (Figure $2 \mathrm{a}$ and $2 \mathrm{~b}$ ). 
The present results demonstrate for the first time the expression of Tie1 in the endothelium of CC in Rat and Human, which appears to exhibit an age-dependent up-regulation. Ongoing research employing molecular studies will clarify this aspect.

\section{References}

1.Cordeiro A.L. et al., Microsc. Microanal., 16: 1- 11, 2010.

2.Figueiredo A. et al.,J. Sex. Med., 8:1341-1351, 2011.

3.Yuan H.T. et al., Mol. Cell Biol., 29:2011-2022, 2009.

4.Yuan H.T. et al., FASEB J., 21:3171-3183, 2007.

5.Patan S., Microvasc. Res., 56:1-21, 1998.
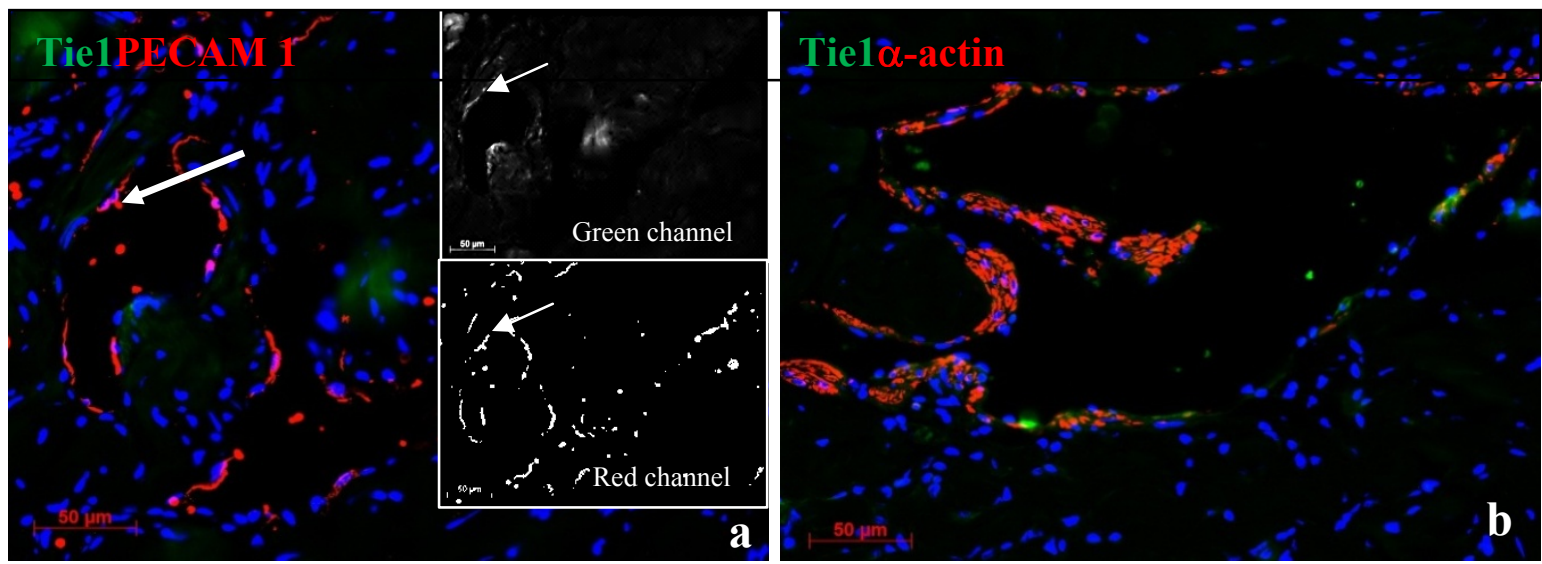

Fig. 1 - Dual immunolabeling of Tie1 (green) and PECAM-1 (red) (Fig. 1a - exposure time $226 \mathrm{~ms}$ ), and $\alpha$ actin (red) (Fig. $1 \mathrm{~b}$ - exposure time $178 \mathrm{~ms}$ ) in corpus cavernosum of Rat aged 24 months. Note co-localization in the endothelium (white arrow) absent in smooth muscle layer (Fig. 1b). Separate labelling of green and red channels (Tie1 and PECAM-1, respectively) shown in Fig. 1A.

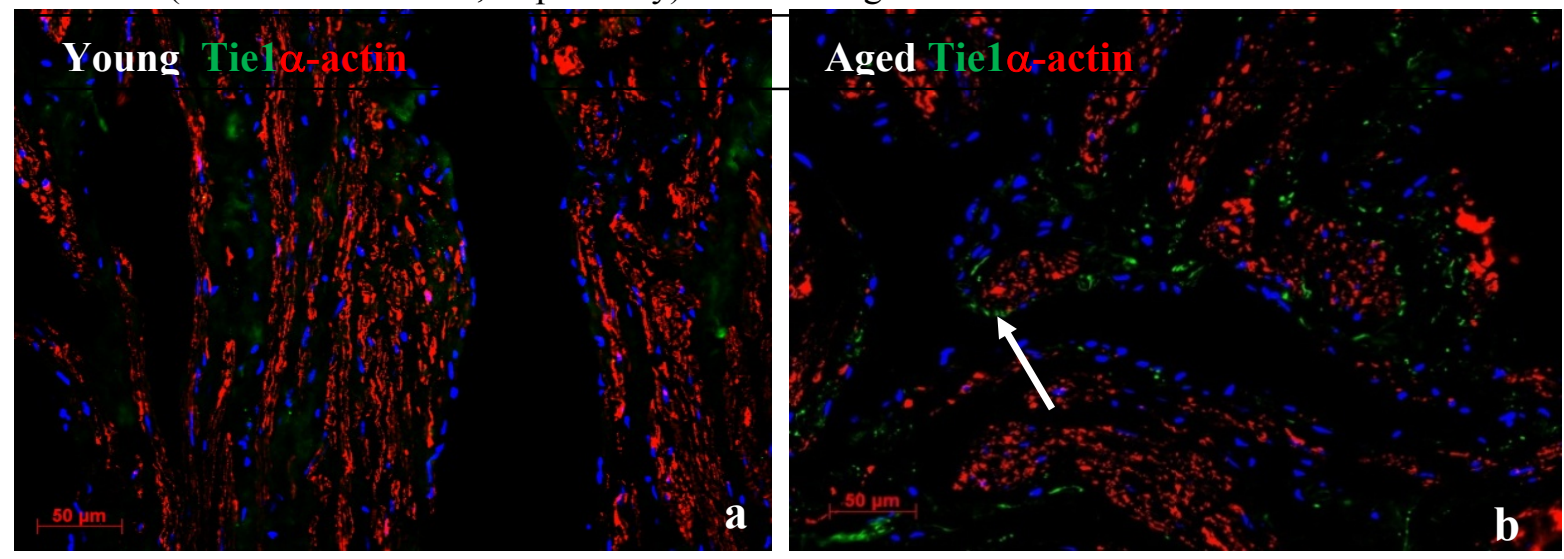

Fig. 2 - Dual immunolabeling of Tie1 (green) and $\alpha$-actin (red) in corpus cavernosum of young (Fig. 2a exposure time $191 \mathrm{~ms}$ ) and aged (Fig. $2 \mathrm{~b}$ - exposure time $109 \mathrm{~ms}$ ) Human. In both conditions, Tie 1 did not label smooth muscle cell, but its expression in the endothelium of the old individual appeared up-regulated (white arrow). 